\title{
Percurso metodológico apoiado por software para mapeamento de indicadores qualitativos para as Boas Práticas em Saúde à população em situação de rua
}

\section{Lucimara Fabiana Fornari', Emiko Yoshikawa Egry', Paula Hino², Carmen Santana $^{2}$ e Elda Oliveira ${ }^{2}$}

${ }^{1}$ Departamento de Enfermagem em Saúde Coletiva, Escola de Enfermagem da Universidade de São Paulo, Brasil | lucimarafornari@usp.br; emiyegry@usp.br | https://orcid.org/0000-0003-0974-0315; https://orcid.org/0000-0002-8655-6549

${ }^{2}$ Escola Paulista de Enfermagem da Universidade Federal de São Paulo, Brasil | paulahino@yahoo.com.br; carmen.santana4370@gmail.com; eldadeoliveira@gmail.com | https://orcid.org/0000-0002-1408-196X; https://orcid.org/0000-0002-6148-6230; https://orcid.org/0000-0002-9973-0948

Resumo: Introdução: A população em situação de rua é um grupo social que demanda atenção especial dos serviços, pois suas necessidades em saúde requerem um cuidado qualificado e atento em decorrência das profundas vulnerabilidades sociais. É primordial o mapeamento de indicadores qualitativos para as Boas Práticas em Saúde (BPS) a essa população. Objetivos: Conhecer as potencialidades e os limites do webQDA para análise qualitativa de uma revisão de escopo sobre o mapeamento de indicadores qualitativos para as BPS à população em situação de rua. Métodos: Trata-se de um estudo qualitativo, integrante de um projeto maior acerca da construção de indicadores de BP na Atenção Primária em Saúde. É baseado na experiência das autoras relacionada à utilização do software webQDA como apoio para a análise qualitativa dos dados coletados por meio da revisão de escopo. Os 29 artigos selecionados na revisão de escopo foram submetidos à análise de conteúdo temática para o mapeamento dos indicadores qualitativos. Resultados: Os artigos na íntegra no formato pdf. foram inseridos como fontes internas no webQDA. O Sistema das Fontes permitiu o armazenamento dos dados provenientes da coleta de forma organizada e com fácil acesso às informações. Contudo, não foi possível realizar a codificação dos textos cujos arquivos apresentavam duas colunas. Optou-se por utilizar instrumento no formato xlsx., inserido por meio da codificação direta. Esse processo otimizou o tempo de trabalho, pois os dados relativos à caracterização dos artigos foram codificados automaticamente como códigos descritivos e as demais informações empíricas foram armazenadas no sistema das fontes internas e codificadas através dos códigos árvores. Conclusões: A potencialidade do webQDA foi verificada na otimização das etapas do tratamento e aprofundamento da análise dos dados empíricos para o mapeamento de indicadores qualitativos. Como limitação, dado o formato em colunas dos artigos, necessitou utilizar o formato xlsx.

Palavras-chave: Pessoas em Situação de Rua; Indicadores de Saúde Comunitária; Enfermagem; Pesquisa Qualitativa; Revisão.

Methodological Path Supported by Software for Mapping Qualitative Indicators for Best Health Practices for the Homeless Population

Abstract: Introduction: The homeless population is a social group that demands specia attention from the health services because their needs require qualified and attentive care, due to the profound social vulnerabilities. It is essential to map qualitative indicators for Best Health Practices (BHP) for this population. Goals: To know the potential and limits of webQDA for qualitative analysis of a scope review on the mapping of qualitative indicators for BHP to the homeless population. Methods: This is a qualitative study, part of a larger project about the construction of BHP indicators in Primary Health Care. It is based on the authors experience related to the use of the webQDA software as a support for the qualitative analysis of the data collected by scope review. The 29 articles selected in the scope review were subjected to thematic content analysis to map qualitative indicators. Results: Articles in full pdf. format were inserted as internal sources in webQDA. The System of Sources allowed the storage of data from the collection in an organized manner and with easy access to information. However, it was not possible to carry out the encoding of texts whose files had two columns. We opted to use an instrument in the xlsx. format, inserted through direct coding. This process optimized the working time, since the data related to the characterization of the articles were automatically coded as descriptive codes and the other empirical information was stored in the system of the internal sources and coded through the tree codes. Conclusions: The potential of webQDA was verified in the optimization of the treatment steps and in-depth analysis of the empirical data for the mapping of qualitative indicators. As a limitation, given the two columns format of the articles, it was necessary to use the xlsx. format.

Keywords: Homeless Persons; Community Health Indicators; Nursing; Qualitative Research Review. 


\section{Introdução}

A população em situação de rua (PSR) tem maiores taxas de morbidade e mortalidade quando comparada com a população em geral. Essas taxas estão associadas ao uso de drogas lícitas e ilícitas e transtornos mentais. Com isso, ocorre a maior demanda aos serviços de saúde para tratamento das condições agudas e crônicas (Fazel, Geddes, \& Kushel, 2014).

O acesso aos serviços de saúde pela PSR é dificultado devido ao estigma, promovendo piora das condições de vida. A PSR é um grupo social situado abaixo da linha da pobreza, tendo como importante marca a exclusão social. No Brasil, nota-se o aumento da população que ocupa as ruas como espaço de abrigo e moradia (Teixeira, Belmonte, Engstrom, \& Lacerda, 2019).

$\mathrm{Na}$ vigência da pandemia do novo Coronavírus, houve um aumento da PSR brasileira, tendo sido acrescido de famílias que perderam sua renda e moradia. O auxílio emergencial dado pelo Governo Federal, pode ter reduzido este contingente de novas famílias em situação de rua. Entretanto, ao término do auxílio, estima-se que novamente houve aumento expressivo da PSR.

O crescimento da PSR, por diversos motivos, demanda a diversificação dos modos de fornecer assistência qualificada nos diferentes níveis de atenção à saúde. Essa assistência tanto pode ser considerada como uma Boa Prática, quanto orientada por outras Boas Práticas em Saúde. Essas Boas Práticas estão relacionadas à melhor forma de identificar, implementar e avaliar as informações produzidas no contexto da saúde, assim como monitorar os resultados das intervenções no cuidado à saúde de usuários ou grupos populacionais. Além disso, consideram as melhores evidências ligadas à segurança, eficácia, efetividade, custo, adequação, valores sociais e éticos, e qualidade, o que resulta no aperfeiçoamento do cuidado à população (Perleth, Jakubowski, \& Busse, 2001).

A literatura registra uma série de práticas realizadas junto à PSR, todavia pouco se sabe se essas práticas de fato resultam no qualitativo "boas", que no presente estudo são entendidas como aquelas que utilizam indicadores de efetividade e avaliação do efeito produzido pelas intervenções no cuidado à saúde.

Os indicadores consistem em parâmetros quantitativos ou qualitativos que detalham os objetivos de uma proposta de acordo com a condução (avaliação do processo) ou o alcance (avaliação dos resultados). Esses indicadores são utilizados como sinalizadores da realidade, que instrumentalizam e não operam por si mesmos (Minayo, 2009).

Nesta perspectiva, as autoras verificaram a necessidade de mapear os indicadores qualitativos para as Boas Práticas em Saúde à PSR. Dessa forma, optou-se por uma revisão de escopo, pois tem como finalidade mapear evidências, explorar a amplitude e a extensão de um tema na literatura, e informar pesquisas futuras. Também é recomendada para identificar e analisar lacunas do conhecimento a respeito de determinado tópico de estudo (Peters et al., 2020).

A revisão de escopo geralmente envolve a gestão de um grande volume de informações provenientes de bases dados da literatura científica e cinzenta. Diante disso, o emprego de Computer Assisted Qualitative Data Analysis Software (CAQDAS) se mostra como um importante suporte aos revisores para a organização, o tratamento e a ampliação da análise dos dados e, consequentemente, apoiam na produção das evidências científicas.

Estudo salienta a utilidade de softwares específicos para revisão associados aos softwares de análise qualitativa. De acordo com os autores, o uso de software torna a análise dos dados mais gerenciável e transparente, favorece a tomada de decisões, a codificação e a agregação dos temas. No caso dos softwares específicos, apresentam funções e ferramentas que possibilitam a auditoria de todas as etapas de revisão. Enquanto os softwares de análise qualitativa, apoiam a análise dos dados e a produção do relatório final (Soilemezi \& Linceviciute, 2018). 
As autoras deste estudo optaram pelo uso do software Web Qualitative Data Analysis (webQDA), devido à experiência na utilização desse CAQDAS. O webQDA é orientado para a análise de dados qualitativos de maneira individual e colaborativa. Permite ao pesquisador editar, visualizar, interligar e organizar os dados, assim como criar categorias, codificar, controlar, filtrar, buscar e questionar os dados (Minayo \& Costa, 2019).

Diante do exposto a pesquisa teve como questão norteadora: Quais as potencialidades e os limites do webQDA para análise qualitativa de dados gerados em uma revisão de escopo? O objetivo foi conhecer as potencialidades e os limites do webQDA para análise qualitativa de uma revisão de escopo sobre o mapeamento de indicadores qualitativos para as Boas Práticas em Saúde à PSR.

\section{Metodologia}

Trata-se de um estudo qualitativo, integrante de um projeto de maior âmbito que pretende a construção de indicadores de Boas Práticas na Atenção Primária em Saúde na perspectiva da Enfermagem em Saúde Coletiva. A primeira fase dele tratou-se de uma revisão de escopo de artigos originais de base empírica para levantar os estudos nacionais e internacionais acerca dos indicadores de efetividade das intervenções junto à PSR1.

Esta pesquisa é baseada na experiência das autoras na utilização do software webQDA como apoio para a análise qualitativa dos dados coletados por meio da revisão de escopo.

O protocolo de revisão foi baseado no processo de revisão de escopo proposto pelo Instituto Joanna Briggs. Ele orientou a procura de estudos que atendessem aos critérios de elegibilidade: estudos publicados em língua inglesa, espanhola e portuguesa, sem delimitação do período de publicação; pesquisas primárias, empíricas, quantitativas e qualitativas de qualquer desenho ou metodologia. Os critérios de inclusão foram estudos que apontavam indicadores ou meios de avaliação de uma prática de cuidado à PSR; estudos que lidavam com avaliação de saúde da PSR decorrente de alguma intervenção; estudos que descreveram uma prática e sua avaliação do ponto de vista da mudança do perfil de saúde ou da condição pré-existente; e estudos que apontavam para o efeito de uma dada prática para a PSR. O critério de exclusão constituiu-se de estudos que apresentam a percepção dos profissionais de saúde sobre uma prática de cuidado a esta população específica.

A questão de revisão foi: Quais indicadores são utilizados para descrever as Boas Práticas em Saúde à PSR? Essa questão foi construída por meio da estratégia PCC, que definiu os elementos: P - População (população em situação de rua); C - Conceito (indicadores de Boas Práticas); C - Contexto (saúde).

A busca dos artigos nas bases de dados foi realizada em dezembro de 2019 e atualizada em agosto de 2020. A coleta de dados foi realizada nas bases de dados que apresentavam interface multidisciplinar sobre o objeto de pesquisa. As bases de dados consultadas foram: Medical Literature Analysis and Retrieval System Online via PubMed (MEDLINE/PubMed), Literatura Latino-Americana e do Caribe em Ciências da Saúde (LILACS), PsycINFO, Cumulative Index to Nursing and Allied Health Literature (CINAHL), Scopus e Excerpta Medical Database (EMBASE). Também foi acessada a biblioteca virtual Scientific Electronic Library Online (SciELO) como fonte adicional.

${ }^{1}$ Hino, P., Fornari, L. F., Santana, C., Oliveira, E., \& Egry, E. Y. (2021). Indicadores de Boas Práticas em Saúde para a população em situação de rua: revisão de escopo. Submetido à publicação. 
Para a busca foram definidos e utilizados os seguintes termos de pesquisa: homeless persons, homeless, runway, foster care, street people, health status indicators, primary care nursing e primary health care. A estratégia de busca foi adotada de acordo com a especificidade de cada base de dados.

A seleção dos estudos de acordo com o título e o resumo foi realizada por meio do aplicativo Rayyan (Ouzzani, Hammady, Fedorowicz, \& Elmagarmid, 2016). Enquanto a seleção dos artigos completos foi realizada por meio do software webQDA (Costa, Moreira, \& Souza, 2019), que também deu suporte para o tratamento e a análise dos dados. Este estudo adotou a técnica de análise de conteúdo temática, que prevê cinco etapas: préanálise, exploração do material, tratamento dos resultados, interpretação e inferência (Bardin, 2011).

A técnica de análise de conteúdo temática foi adaptada ao webQDA. Este software é acessível pela internet, tem compatibilidade com diversos sistemas operacionais e permite o compartilhamento de informações entre os utilizadores (Freitas et al., 2017). Neste estudo, a primeira autora participou como gestora do projeto, a segunda autora como colaboradora e as demais como convidadas.

O software foi incorporado ao processo de análise qualitativa dos dados provenientes da revisão de escopo com a finalidade de organizar e sistematizar as informações, devido à sua flexibilidade no gerenciamento dos dados e na configuração dos resultados (Costa \& Amado, 2018).

O webQDA está estruturado em quatro sistemas: Fontes (inserção dos dados), Codificação (criação das categorias descritivas e empíricas), Questionamento (cruzamento dos conjuntos de dados) e Gestão (organização do fluxo de trabalho individual e colaborativo). O Sistema de Fontes está dividido em internas, externas e notas. O Sistema de Codificação dispõe de códigos livres e árvore, descritores e classificações. O Sistema de Questionamento tem palavras mais frequentes, pesquisa de texto ou código, e matrizes. O Sistema de Gestão apresenta a inclusão dos utilizadores, o diário de bordo, o fluxo de trabalho e os registros.

\section{Resultados e Discussão}

Apesar de o webQDA dispor de funcionalidade específica para o desenvolvimento de revisões da literatura, neste estudo as autoras optaram por utilizar o aplicativo Rayyan no processo de triagem inicial dos títulos e resumos.

O Rayyan é um aplicativo gratuito para web que otimiza o processo de triagem inicial e a colaboração entre os revisores. Caracteriza-se pela facilidade de acesso às pesquisas, pela economia de tempo e pela simplicidade no compartilhamento e na comparação das decisões entre os revisores (Ouzzani et al., 2016).

A buscas realizadas nas sete bases de dados foram salvas no formato de arquivo BibTex e importadas para o aplicativo Rayyan. O aplicativo identificou automaticamente 254 artigos duplicados. Posteriormente, duas das autoras desse estudo realizaram a leitura de maneira independente e duplo cega dos 761 títulos e resumos selecionados. Em seguida, uma terceira autora realizou a revisão dos artigos que apresentaram divergência na decisão para inclusão ou exclusão do estudo (Fig. 1). Nos casos em que a dúvida sobre a seleção permaneceu, os artigos avançaram para a etapa seguinte correspondente à leitura na íntegra. 


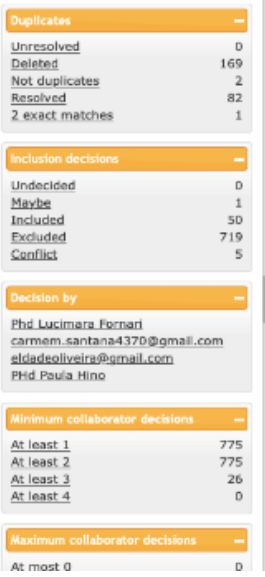

2019-12-11: Indicadores de boas práticas de enfermagem na APS referentes a PSR

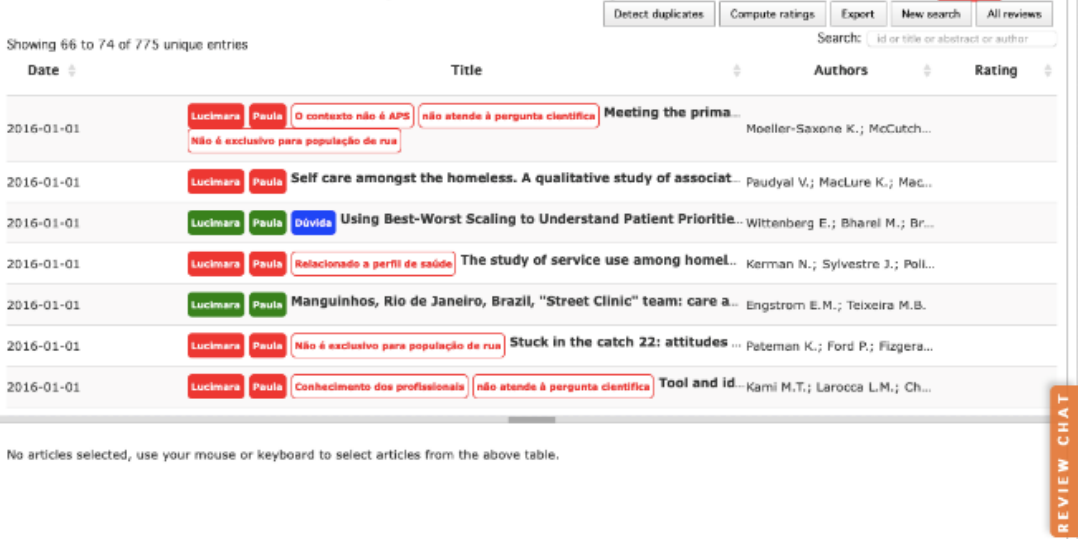

Fig. 1. Processo de triagem inicial dos títulos e resumos no aplicativo Rayyan.

Foram selecionados 82 artigos na íntegra e importados no formato Portable Document Format para o webQDA por meio do sistema de Fontes Internas. Contudo, no início do tratamento dos dados verificou-se que o software não permitia codificar os arquivos que tinham duas colunas. Diante disso, as autoras optaram por realizar a extração dos dados dos artigos na íntegra por meio de instrumento elaborado de acordo com a pergunta de revisão (Fig. 2), no qual foram verificados os seguintes itens: ano de publicação, área de concentração, país da instituição que produziu o artigo, tipo de estudo, amostra do estudo, local do estudo, ação realizada, indicador de qualidade, meio de avaliação, resultado ou desfecho encontrado, benefícios do ponto de vista dos usuários e dos autores do estudo.

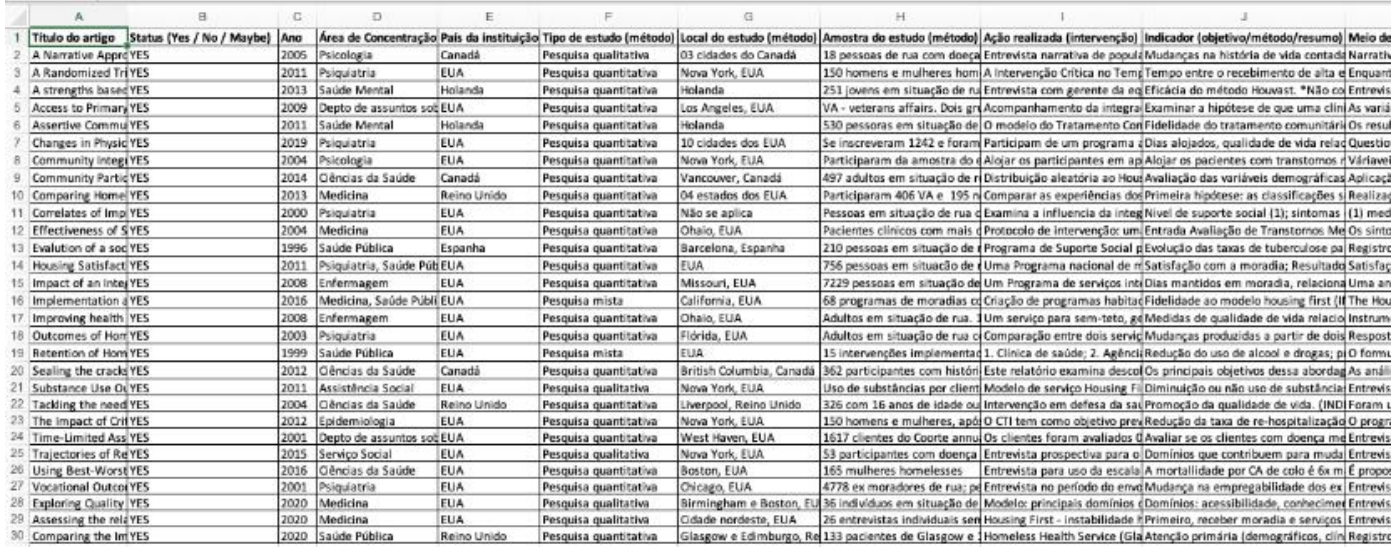

Fig. 2. Instrumento de extração dos dados dos artigos na íntegra.

Quatro autoras do estudo ficaram responsáveis pela leitura de 16 artigos cada e uma autora ficou responsável pela leitura de 18 artigos. Após a leitura de todos os artigos foi marcada uma reunião online através da Plataforma Google Meet, na qual cada artigo foi apresentado seguido pela justificativa da inclusão ou exclusão. Nesta etapa da revisão foram excluídos 56 artigos.

Devido ao tempo decorrido entre a busca dos artigos nas bases de dados e a triagem dos artigos completos, verificou-se a necessidade de atualização da busca oito meses após a primeira, sendo rastreados 11 artigos elegíveis para a revisão de escopo. Esses artigos foram lidos na íntegra por duas autoras do estudo de forma independente. Posteriormente, houve o agendamento de uma nova reunião online com o grupo das autoras do estudo para a definição da amostra final (29 artigos). 
O instrumento preenchido com as informações dos 29 artigos foi incorporado ao webQDA. Para tanto, foi utilizada a funcionalidade de codificação direta, na qual os dados correspondentes à caracterização dos artigos (ano de publicação, área de concentração, país da instituição que produziu o artigo, tipo de estudo, amostra do estudo e local do estudo) foram codificados automaticamente como códigos descritivos (Fig. 3).

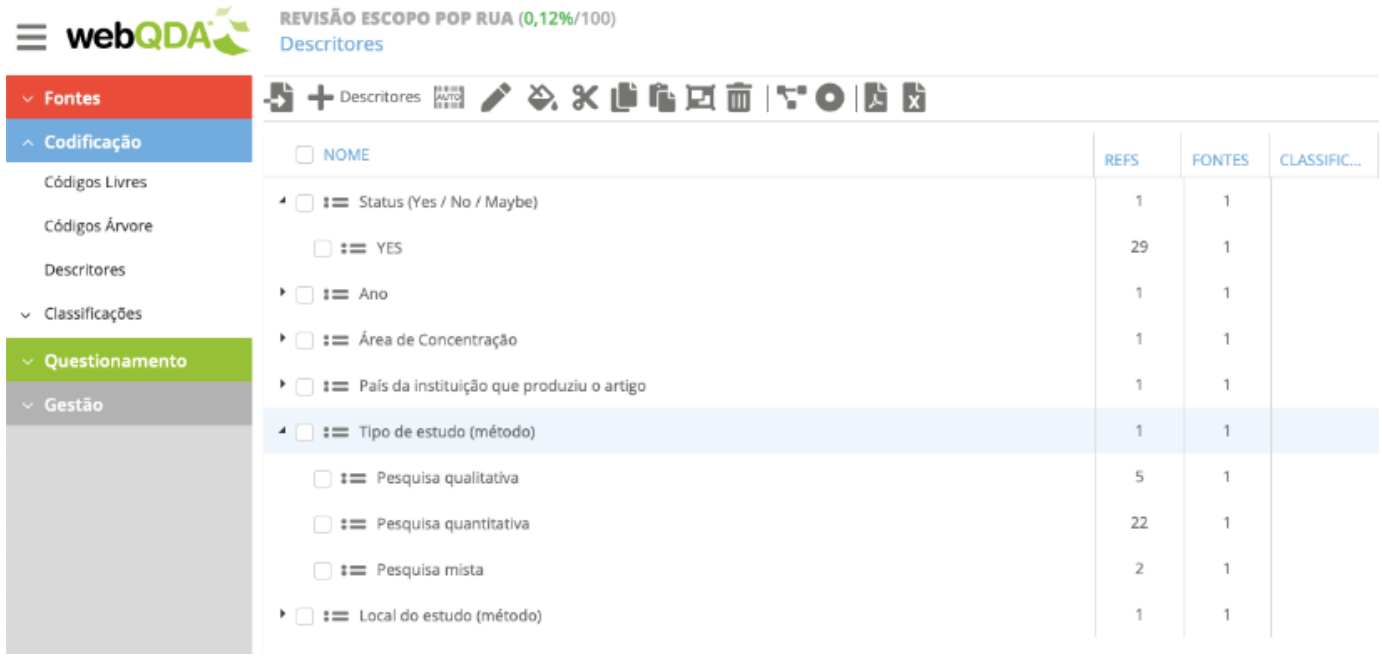

Fig. 3. Códigos descritivos associados à revisão de escopo.

As demais informações associadas aos dados empíricos dos artigos (ação realizada, indicador de qualidade, meio de avaliação, resultado ou desfecho encontrado, benefícios do ponto de vista dos usuários e dos autores do estudo) foram importadas para o sistema de Fontes Internas e submetidas à técnica de análise de conteúdo temática.

No webQDA, as categorias empíricas foram construídas por meio dos códigos árvores (Fig. 4). A primeira autora do estudo realizou a codificação de todos os dados e a segunda autora realizou a validação por meio da funcionalidade da codificação oculta.

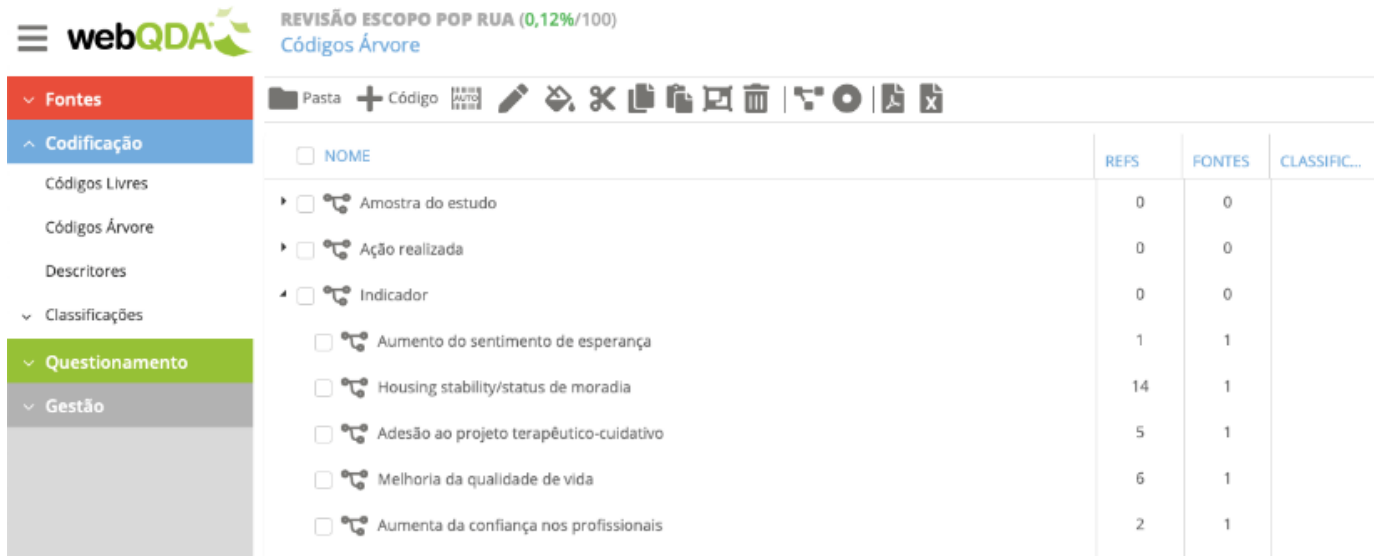

Fig. 4. Códigos árvores associados à revisão de escopo.

Os indicadores qualitativos, correspondentes à cada artigo selecionado, foram agrupados em quatro categorias empíricas: Relação do usuário com os serviços de atendimento; Avaliação das condições de saúde e doença; Avaliação da inclusão social e Avaliação de mudanças nas características comportamentais e psicológicas (Quadro 1). 
Tabela 1. Descrição das categorias de indicadores e seus respectivos indicadores.

\begin{tabular}{|c|c|}
\hline $\begin{array}{l}\text { Categorias de } \\
\text { indicadores }\end{array}$ & Indicadores \\
\hline $\begin{array}{l}\text { Relação do usuário com } \\
\text { o serviço de } \\
\text { atendimento }\end{array}$ & $\begin{array}{l}\text { Acesso e uso dos serviços de saúde; Número e frequência de } \\
\text { internação hospitalar ou psiquiátrica; Adesão ao projeto } \\
\text { terapêutico-cuidativo; Aumento da confiança nos profissionais; } \\
\text { Working aliance e relação médico-paciente; Assistência jurídica } \\
\text { e sistema de justiça; Motivos para permanência e saída do } \\
\text { programa; Qualidade do cuidado primário; Melhoria do acesso } \\
\text { ao cuidado; Experiências nos serviços. }\end{array}$ \\
\hline $\begin{array}{l}\text { Avaliação das condições } \\
\text { de saúde e doença }\end{array}$ & $\begin{array}{l}\text { Frequência e intensidade do consumo de álcool, tabaco e outras } \\
\text { substâncias psicoativas; Melhoria da saúde física, mental e } \\
\text { sexual; Melhoria da qualidade de vida; Avaliação dos } \\
\text { transtornos mentais, condições médicas e doenças infeciosas; } \\
\text { Efetividade das condições médicas; Evolução das taxas de } \\
\text { tuberculose. }\end{array}$ \\
\hline $\begin{array}{l}\text { Avaliação de inclusão } \\
\text { social }\end{array}$ & $\begin{array}{l}\text { Housing stability, estado de moradia, tempo sem teto e } \\
\text { satisfação com a moradia; Participação social e melhoria nos } \\
\text { relacionamentos interpessoais e com a comunidade; Suporte e } \\
\text { apoio social; Emprego e renda. }\end{array}$ \\
\hline $\begin{array}{l}\text { Avaliação de mudanças } \\
\text { nas características } \\
\text { comportamentais e } \\
\text { psicológicas }\end{array}$ & $\begin{array}{l}\text { Necessidades de cuidados psicológicos e específicas dos } \\
\text { moradores de rua; Satisfação geral com a vida e saúde, } \\
\text { resultados subjetivos e funcionais; Escolhas e mudanças de } \\
\text { vida; Sensação de segurança e proteção; Comportamento de } \\
\text { busca de ajuda e funcionamento mental e social; Coping; } \\
\text { Resiliência; Vitimização; Sentimento de esperança; } \\
\text { Incapacidade intelectual; Modelo comportamental; Recuperação } \\
\text { clínica, existencial, funcional, física e social; Gestão das } \\
\text { condições agudas e crônicas. }\end{array}$ \\
\hline
\end{tabular}

O quadro contendo a síntese dos indicadores qualitativos para as Boas Práticas em Saúde à PSR foi validado pelas demais autoras do estudo considerando sua expertise no tema da revisão de escopo.

Compreende-se que a revisão envolve um processo de trabalho complexo e árduo, que se mostra desafiador diante da necessidade de coerência interna, abrangência na busca de estudos, transparência e rigor em todas as etapas de desenvolvimento. Assim, ressaltase a importância de utilizar um software para dar suporte para a organização das etapas de revisão e dos achados, de forma a obter sínteses coerentes e robustas, que contribuam para a produção de conhecimentos (Soares, 2019).

O processo de revisão pode ser otimizado por meio da utilização de softwares que apoiam a organização, o tratamento e a análise dos dados. Eles também reforçam a adequação das etapas do percurso metodológico e promovem credibilidade às evidências científicas (Fornari \& Pinho, 2019). Dessa forma, considera-se que os softwares potencializam a construção das revisões por meio da organização dos dados, e influenciam no tempo de duração, na transparência metodológica e no trabalho colaborativo entre os membros da equipe de investigação (Dixon-Woods, Fitzpatrick, \& Roberts, 2001; Grant \& Booth, 2009).

Revisão da literatura sobre o uso de ferramentas digitais como apoio para o desenvolvimento de revisões, verificou que as ferramentas específicas foram fundamentais na etapa de seleção dos documentos e no tratamento dos dados. Contudo, apesar de serem específicas para revisões, não foram utilizadas em todas as etapas metodológicas. No que se refere às ferramentas de análise qualitativa, foram primordiais na etapa de tratamento e síntese dos dados (Fornari, Pinho, de Almeida, \& Costa, 2019).

$\mathrm{Na}$ presente revisão, as ferramentas digitais de apoio foram utilizadas em todas as etapas da metodologia, com exceção do momento de elaboração do protocolo de revisão. A experiência das autoras revela que o processo de revisão foi otimizado diante da facilidade de gerenciamento dos dados por meio das ferramentas, que possibilitou maior tempo para a produção das análises e reflexões sobre os indicadores qualitativos para as Boas Práticas em Saúde à PSR. 
Estudo realizado a partir de uma revisão sistemática sobre o impacto dos softwares de análise qualitativa na reflexividade dos investigadores, verificou que os softwares foram responsáveis pelo aprimoramento da coleta, exploração e codificação dos dados. A utilização desses softwares também potencializou as ações, análises e interpretações dos investigadores (Woods, Macklin, \& Lewis, 2016).

Por fim, a partir da experiência das pesquisadoras na condução da presente revisão, considera-se que o percurso de revisão é influenciado por diferentes aspectos, como: pelo propósito e pela experiência dos investigadores em relação ao método e tema de investigação, pela quantidade e qualidade das publicações, pelo acesso e domínio dos recursos, pela perspectiva teórico e metodológica, e pelo tempo de desenvolvimento.

\section{Conclusões}

O software webQDA se mostrou potente para análise dos dados produzidos a partir de uma revisão de escopo, uma vez que apoiou a organização dos dados por meio da importação direta dos metadados extraídos das bases de dados de maneira sincronizada, ampliou o tratamento e aprofundou a análise do material empírico devido à possibilidade de apresentar as sínteses do conhecimento através do formato de texto, tabela e figura. Além disso, o fato de estar disponível na web favoreceu o trabalho colaborativo entre as autoras do estudo pertencentes à duas instituições de ensino superior distintas. Em época de distanciamento social, o software facilitou grandemente o desenvolvimento do estudo, especialmente na parte de análise e discussão. A participação de mais de um revisor no mesmo projeto favoreceu a credibilidade na seleção das publicações e na codificação dos resultados, uma vez que diminuiu o viés interpretativo. Os limites encontrados foram os relacionados à decodificação e análise do material empírico, os artigos em pdf., que quando dispostos em duas colunas necessitou a conversão em xlsx.

O estudo ressaltou o papel do webQDA unicamente como apoio para a análise dos dados empíricos, pois esses não são automaticamente codificados pelo software, mas requerem o conhecimento dos pesquisadores a respeito da técnica de análise dos dados proposta na metodologia, ao processo de desenvolvimento da revisão de escopo e à aproximação com o tema objeto de estudo.

\section{Agradecimentos}

Agradecemos à Bibliotecária-Chefe da Universidade Federal de São Paulo Campus São Paulo Dra. Andreia do Carmo pelo apoio na busca dos dados. Esta pesquisa é resultado de projeto financiado por Bolsa de Produtividade em Pesquisa do CNPq, por Bolsa do Programa Nacional de Pós-Doutorado da CAPES, e pelo CNPq - Processo 408589/20183.

\section{Referências}

Bardin, L. (2011). Análise de conteúdo. São Paulo: Edições 70.

Blismas, N. G., \& Dainty, A. R. J. (2003). Computer-aided qualitative data analysis: Panacea or paradox? Building Research and Information, 31(6), 455-463. https://doi.org/10.1080/0961321031000108816

Costa, A. P., \& Amado, J. (2018). Content Analysis Supported by Software. Aveiro - Portugal: Ludomedia.

Costa, A. P., Moreira, A., \& Souza, F. N. de. (2019). webQDA - Qualitative Data Analysis. Aveiro Portugal: Aveiro University and MicrolO. 
Vol. 8 | Investigação Qualitativa em Saúde: Avanços e Desafios

Fazel, S., Geddes, J. R., \& Kushel, M. (2014). The health of homeless people in high-income countries: Descriptive epidemiology, health consequences, and clinical and policy recommendations. The Lancet, 384(9953), 1529-1540. https://doi.org/10.1016/S01406736(14)61132-6

Freitas, F., Ribeiro, J., Brandao, C., Reis, L. P., Souza, F. N. de, \& Costa, A. P. (2017). Learn by yourself: The Self-Learning Tools for Qualitative Analysis Software Packages. Digital Education Review, (32), 97-117.

Minayo, M. C. de S. (2009). The construction of qualitative indicators for the evaluation of changes. Revista Brasileira de Educação Médica, 33(Supl. 1), 83-91.

Minayo, M. C. de S., \& Costa, A. P. (2019). Techniques that use speech, observation and empathy: qualitative research in action. Aveiro - Portugal: Ludomedia.

Ouzzani, M., Hammady, H., Fedorowicz, Z., \& Elmagarmid, A. (2016). Rayyan-a web and mobile app for systematic reviews. Systematic Reviews, 5(1). https://doi.org/10.1186/s13643-0160384-4

Perleth, M., Jakubowski, E., \& Busse, R. (2001). What is "best practice" in health care? State of the art and perspectives in improving the effectiveness and efficiency of the European health care systems. Health Policy, 56(3), 235-250. https://doi.org/10.1016/S0168-8510(00)00138$\mathrm{X}$

Peters, M., Godfrey, C., Mcinerney, P., Munn, Z., Tricco, A., \& Khalil, H. (2020). Chapter 11: Scoping Reviews. In E. Aromataris \& Z. Munn (Eds.), JBI Manual for Evidence Synthesis.

Soilemezi, D., \& Linceviciute, S. (2018). Synthesizing Qualitative Research: Reflections and Lessons Learnt by Two New Reviewers. International Journal of Qualitative Methods, 17(1), 1-14. https://doi.org/10.1177/1609406918768014

Teixeira, M. B., Belmonte, P., Engstrom, E. M., \& Lacerda, A. (2019). The invisible urban dwellers: the stigma of People Living in the Streets in Rio de Janeiro. Saúde Em Debate, 43(7), 92 101. https://doi.org/10.1590/0103-11042019s707 